\title{
Probing the Mass Fraction of MACHOs in Extragalactic Halos
}

\section{Citation}

Perna, Rosalba, and Abraham Loeb. 1998. "Probing the Mass Fraction of MACHOs in

Extragalactic Halos." The Astrophysical Journal 493 (2): 523-28. https://doi.org/10.1086/305170.

\section{Permanent link}

http://nrs.harvard.edu/urn-3:HUL.InstRepos:41393228

\section{Terms of Use}

This article was downloaded from Harvard University's DASH repository, and is made available under the terms and conditions applicable to Other Posted Material, as set forth at http:// nrs.harvard.edu/urn-3:HUL.InstRepos:dash.current.terms-of-use\#LAA

\section{Share Your Story}

The Harvard community has made this article openly available.

Please share how this access benefits you. Submit a story.

\section{Accessibility}


The AstrophysiCAL Journal, 493:523-528, 1998 February 1

(C) 1998. The American Astronomical Society. All rights reserved. Printed in U.S.A.

\title{
PROBING THE MASS FRACTION OF MACHOs IN EXTRAGALACTIC HALOS
}

\author{
Rosalba Perna AND Abraham Loeb \\ Harvard-Smithsonian Center for Astrophysics, 60 Garden Street, Cambridge, MA 02138 \\ Received 1997 June 12; accepted 1997 September 10
}

\begin{abstract}
Current microlensing searches calibrate the mass fraction of the Milky Way halo that is in the form of massive astrophysical compact halo objects (MACHOs). We show that surveys like the Sloan Digital Sky Survey (SDSS) can probe the same quantity in halos of distant galaxies. Microlensing of background quasars by MACHOs in intervening galaxies would distort the equivalent width distribution of the quasar emission lines by an amplitude that depends on the projected quasar-galaxy separation. For a statistical sample of $\sim 10^{5}$ quasars as expected in the SDSS, this distortion is detectable at the $\gtrsim 2 \sigma$ level out to a quasar-galaxy impact parameter of several tens of $\mathrm{kpc}$, as long as extragalactic halos are made of MACHOs. Detection of this signal would test whether the MACHO fraction inferred for the Milky Way halo is typical of other galaxies.

Subject headings: dark matter — galaxies: halos — gravitational lensing — quasars: general
\end{abstract}

\section{INTRODUCTION}

One of the basic unknowns in cosmology is the fraction of the dark matter mass that is in the form of compact objects, such as stellar remnants. Paczyński (1986) proposed monitoring of background stars in the Large Magellanic Cloud (LMC) and searching for rare amplification events due to microlensing as a means of constraining the mass fraction of compact objects in the Milky Way halo extending to $50 \mathrm{kpc}$. Current microlensing surveys suggest that $\sim 50+20 \%$ of the mass of the halo of the Milky Way Galaxy might be in the form of massive astrophysical compact halo objects (MACHOs) with a mass $\sim 0.5_{-0.2}^{+0.3} M_{\odot}$ (Alcock et al. 1996). It remains to be seen whether future studies confirm these early reports.

The search for brightening of individual background stars due to microlensing by foreground MACHOs cannot be trivially extended beyond the scale of the Milky Way halo. Images of galaxies at large distances include many faint stars within each resolution element (pixel), most of which are unlensed. Alternatively, one can search for a weak temporal fluctuation due to the chance amplification of one of the stars within a given pixel (Gould 1996; Crotts 1996). This method is currently being applied to the nearest massive galaxy, M31 (Crotts \& Tomaney 1996).

At yet larger distances, MACHOs can be detected by their effect on quasars. The lensing cross section of a MACHO increases in proportion to its distance from the observer. Thus, despite the fact that quasars are rare, the likelihood of a quasar that happens to be located behind an extragalactic halo to be microlensed is $\sim 5-6$ orders of magnitude larger than that of an LMC star behind the Galactic halo. This makes quasars useful sources for the study of extragalactic halos as LMC stars are for the Galactic halo. The only question is how one can identify the existence of microlensing in the former case.

The crossing time of the lensing zone of solar mass MACHOs in the Galactic halo is of the order of a month, and so microlensing of LMC stars can be conveniently identified through the achromatic variability signal of an otherwise steady stellar source. However, if the same halo objects are placed at cosmological distances, the duration of their lensing event is stretched by $\sim 2-3$ orders of magnitude up to the inconvenient scale of decades. Moreover, the lensing events of quasars would be contaminated by noise from the uncertain level of intrinsic variability of the quasars on the same timescale (Maoz 1996). Therefore, variability is not an efficient search technique for microlensing by solar mass MACHOs in distant halos. ${ }^{1}$

An alternative method that does not require monitoring over time is to search for a change in the equivalent width of the broad emission lines of the quasar. The characteristic Einstein radius of a solar mass lens at a cosmological distance is $\sim 5 \times 10^{16} \mathrm{~cm}$, comfortably between the scales of the continuum-emitting accretion disk $\left(\lesssim 10^{15} \mathrm{~cm}\right)$ and the broad-line region $\left(\sim 3 \times 10^{17} \mathrm{~cm}\right)$ of a bright quasar. This implies that a microlensing event would significantly amplify the continuum but not the broad lines emitted by the quasar. As a result, the equivalent width distribution of the broad emission lines (Francis 1992) will be systematically distorted in a sample of microlensed quasars (Canizares 1982, 1984). Dalcanton et al. (1994) used the lack of redshift evolution in the equivalent width distribution of quasars to limit the mean density parameter of MACHOs with a mass $10^{-3}$ to $60 M_{\odot}$ in the universe, $\Omega_{\text {MACHOs }} \lesssim 0.2$. This limit, however, does not exclude the possibility that MACHOs account for most of the dark matter in galactic halos (or even globally, if the universe is open). Since luminous stars cluster in galaxies, it is possible that their dim counterparts are also concentrated around galaxies.

The probability for microlensing depends on the projected separation between the background quasar and the center of the intervening galaxy. In this paper we propose to look for MACHOs in extragalactic halos, by searching for the equivalent width distortion of quasar emission lines as a function of the projected quasar-galaxy separation. The forthcoming Sloan Digital Sky Survey (SDSS; to be available on-line at Princeton University) will catalog $\sim 10^{5}$ quasars and $\sim 5 \times 10^{7}$ galaxies, and would provide an ideal database for such a study.

The outline of the paper is as follows. In $\S 2$ we describe our model assumptions. In $\S 3$ we derive the expected dis-

\footnotetext{
${ }^{1}$ Although Jupiter mass lenses could cause quasar variability on the more convenient timescale of $\lesssim 1$ yr (Hawkins \& Taylor 1997), current microlensing searches (Alcock et al. 1996; Ansari et al. 1996) rule out the possibility that such objects contribute significantly to the mass of the Galactic halo.
} 
tortion of the equivalent width distribution of background quasars as a function of the MACHO mass fraction in the intervening galactic halos. In $\S 4$ we quantify the expected lensing signal in SDSS, using Monte Carlo realizations of the quasar and galaxy fields. Finally, $\S 5$ summarizes our main conclusions.

\section{MODEL ASSUMPTIONS}

We model a galactic halo as a singular isothermal sphere (SIS), whose average surface mass density at a distance $\xi$ from the center is $\Sigma(\xi)=\sigma^{2} /(2 G \xi)$. The one-dimensional velocity dispersion, $\sigma$, is related to the luminosity, $L$, of the galaxy through the Tully-Fisher (1977) relation, $\sigma / \sigma_{*}=$ $\left(L / L_{*}\right)^{\alpha}$, with $\alpha \approx 0.4$ in the $R$ band (Strauss \& Willick 1995), $\sigma_{*} \approx 170 \mathrm{~km} \mathrm{~s}^{-1}$, and $L_{*}=10^{10} L_{\odot} h^{-2}$. It is convenient to normalize all length scales in the lens plane by

$$
\xi_{0}=4 \pi\left(\frac{\sigma}{c}\right)^{2} \frac{D_{\mathrm{ol}} D_{\mathrm{ls}}}{D_{\mathrm{os}}},
$$

where $D_{\mathrm{ol}}, D_{\mathrm{os}}$, and $D_{\mathrm{ls}}$ are the observer-lens, the observersource, and the lens-source angular diameter distances, respectively. These distances depend on the cosmological parameters; in the following, we assume $\Omega=1, \Lambda=0$, and $h=0.5$. Defining $x=\xi / \xi_{0}$ as the normalized image position in the lens plane, the amplification factor of a point source by a SIS is

$$
\langle\mu\rangle=\frac{x}{|x-1|} .
$$

Multiple images occur when $x<2$. For $x \geq 2$, the single image position is at $x=y+1$, where $y=\eta D_{\mathrm{ol}} /\left(D_{\mathrm{os}} \xi_{0}\right)$, and $\eta$ is the distance of the source from the optical axis in the source plane (Schneider, Ehlers, \& Falco 1992).

Equation (2) yields the amplification factor $\mu$ only in the case where the mass distribution is perfectly smooth. However, in the presence of MACHOs, one obtains a probability distribution of amplification factors at any given value of $x$. The average amplification with this distribution must still be given by equation (2) to guarantee flux conservation. Since the lensing zone of a solar mass MACHO is 6 orders of magnitude smaller than the length scale over which the global $\Sigma$ varies, the MACHOs can be treated locally as a field of randomly distributed point masses with some average surface density $\Sigma$. We then define $p(\mu, x, \epsilon) d \mu$ as the probability that a point source whose line of sight passes at an impact parameter $x$, relative to the center of a galaxy, is amplified by a factor between $\mu$ and $\mu+d \mu$. This probability depends on the MACHO mass fraction $\epsilon$, which we take as a constant. Schneider (1987) and Bartelmann \& Schneider (1990) derived the following analytical expression that agrees reasonably well with results from numerical simulations:

$$
\begin{aligned}
p(\mu, x, \epsilon)= & {\left[\frac{C \times\left(\mu-\mu_{*}\right)^{\eta}}{\mu^{\eta+3}}+A\left(\mu-\mu_{*}\right)\right.} \\
& \times \exp (-B \mu) d \mu] H\left(\mu-\mu_{*}\right) d \mu .
\end{aligned}
$$

Here $H(\ldots)$ denotes the Heaviside step function; $A, B$, and $C$ are functions of $x$. The first two are determined by the constraints of normalization and flux conservation and the third by the limit of high amplification (Schneider 1987). The parameter $\eta$ is chosen so as to make $A$ and $B$ well defined and positive for all possible values of $x$. The value of $\eta$ rises monotonically with decreasing $\epsilon$, because the probability distribution approaches a $\delta$-function as the MACHO fraction goes to zero. The parameter $\mu_{*}$ is a function of $\epsilon$, such that it approaches $\langle\mu\rangle$ in the limit of $\epsilon \rightarrow 0$ (see Bartelmann \& Schneider 1990 for full details).

Our goal is to quantify the distortion of the equivalent width distribution of quasar emission lines due to microlensing by MACHOs in halos of foreground galaxies. For a given magnitude-limited sample of galaxies and quasars, we need to select the galaxy and quasar properties at random. We assume that the local luminosity function of galaxies has the Schechter form,

$$
\phi_{G}(L) d L=\phi_{*}\left(\frac{L}{L_{*}}\right)^{v} \exp \left(-\frac{L}{L_{*}}\right) d\left(\frac{L}{L_{*}}\right),
$$

with $\phi_{*}=0.018(h / 0.5)^{3} \mathrm{Mpc}^{-3}$ and $v=-0.7$ in the $R$ band (Lin et al. 1996). Since a typical survey contains galaxies at very low redshifts (e.g., $\langle z\rangle \sim 0.1$ for the SDSS), we ignore evolutionary corrections and $k$-corrections to this luminosity function and scale the density of galaxies in each luminosity bin by $(1+z)^{3}$. On the other hand, the quasar density peaks at high redshifts $z=2-4$ (see Fig. 2 in Shaver et al. 1996). Because of the disparity between the lens and source redshifts, we can assume that all the quasars are at an effective redshift $z_{s} \approx 3$ and make use of their welldetermined total number count. The inaccuracy introduced by this approximation to the lensing calculation is small $(\sim 10 \%-20 \%)$ and not significant relative to the observational uncertainties in the redshift evolution of the quasar luminosity function. In this approach, we only need to use the integral number counts of quasars as a function of $B$-band flux, which is well described by a broken power law,

$$
N(>S)=C^{\prime} \times \begin{cases}\left(S / S_{0}\right)^{-\beta_{1}} & \text { if } S \leq S_{0}, \\ \left(S / S_{0}\right)^{-\beta_{2}} & \text { if } S>S_{0},\end{cases}
$$

where $\beta_{1} \approx 0.9$ and $\beta_{2} \approx 2.5$ (e.g., Setti 1984; Marshal 1985), and $C^{\prime} \approx 10 \mathrm{deg}^{-2}$ (Hartwick \& Schade 1990). The break flux $S_{0}$ corresponds to a $B$ magnitude $\sim 19.5$. We can safely ignore any correlation between the quasars and the galaxies because of the clear separation between their redshifts.

\section{SIGNATURE OF MICROLENSING}

The flux of a quasar can be significantly amplified by microlensing only if the size of its emission region is smaller than the projected Einstein radius of the lens in the source plane, $r_{\mathrm{E}}$. The maximum amplification of a circular source of radius $r_{s}$ and uniform brightness is given by (see Schneider et al. 1992, p. 38)

$$
\mu_{\max }=\sqrt{1+4\left(r_{\mathrm{E}} / r_{s}\right)^{2}} .
$$

At cosmological distances, the Einstein radius of a star of mass $M_{\text {star }}$ obtains the characteristic value $r_{\mathrm{E}} \sim 5$ $\times 10^{16}\left(M_{\text {star }} / M_{\odot}\right)^{1 / 2} \mathrm{~cm}$. In comparison, the optical continuum emission of quasars is believed to originate from a compact accretion disk. The UV bump observed in quasar spectra is often interpreted as thermal emission from an accretion disk with a surface temperature $T_{\text {disk }} \equiv 10^{5} T_{5} \mathrm{~K}$, where $T_{5} \sim 1$ (e.g., Laor 1990), and so the scale of the disk emission region must be $\sim 10^{15} \mathrm{~cm} \times T_{5}^{-2} L_{46}^{1 / 2}$, where $L_{46}$ is the corresponding $\sim 3 \times 10^{17} \mathrm{~cm} L_{46}^{1 / 2}$ luminosity of the quasar in units of $10^{46} \mathrm{ergs} \mathrm{s}^{-1}$. Thus, for lens masses $M_{\text {star }} \gg 10^{-3} M_{\odot}$, the continuum source is much smaller 


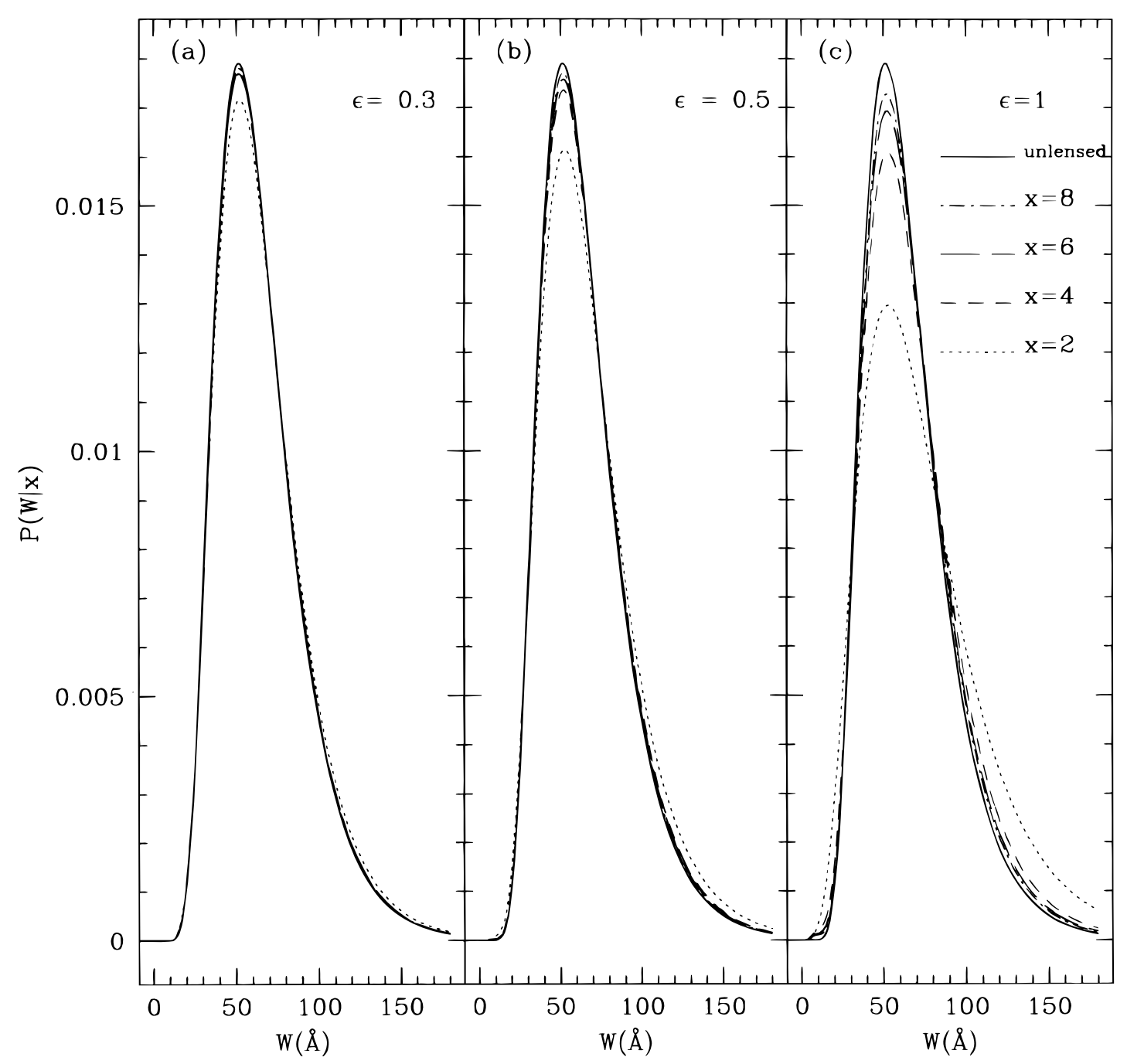

Fig. 1.-EW distribution for the Mg II emission of quasars. The solid line reflects the assumed unlensed distribution. The other curves show the distributions expected for a sample of microlensed quasars whose line of sight passes at an impact parameter $x=2$ (dotted line), $x=4$ (dashed line), $x=6$ (long-dashed line), and $x=8$ (dash-dotted line) from the center of a foreground galactic halo. In (a) the MACHO fraction is $\epsilon=0.3$, in $(b) \epsilon=0.5$, and in (c) $\epsilon=1$.

than the Einstein radius and could therefore be amplified considerably. This expectation is indeed confirmed in the nearby lens of Q2237+0305, where variability due to microlensing has been observed (Wambsganss, Paczyński, \& Schneider 1990; Rauch \& Blandford 1991; Racine 1991; Gould \& Miralda-Escudé 1996). On the other hand, reverberation studies of the time lag between variations in the continuum and the line emission in active galactic nuclei indicate that the broad emission lines of quasars originate at a distance of $\sim 3 \times 10^{17} \mathrm{~cm} L_{46}^{1 / 2}$ (Peterson 1993; Maoz 1996; Netzer \& Peterson 1997). For a solar mass lens and a quasar with $L_{46} \sim 1$, equation (6) implies that the maximum line amplification differs from unity by only $\lesssim 10 \%$. This argues that microlensing of the broad-line region by a single star can be neglected. The broad-line region would, however, be macrolensed by a factor $\langle\mu\rangle$ due to the average effect of the galaxy as a whole. As a result of this differential amplification of lines and continuum, the equivalent width of the lines will change during a microlensing event.

More specifically, let us define $I_{v}\left(\lambda_{0}\right)$ to be the intensity of the quasar continuum in the neighborhood of the wavelength $\lambda_{0}$ of a particular emission line and $\Delta I_{v}(\lambda)$ to be the difference between the total (line + continuum) intensity and the continuum. If the continuum is amplified by a factor $\mu$, then its observed intensity changes to $\mu I_{v}\left(\lambda_{0}\right)$. The intensity of the lines is enhanced by a factor $\langle\mu\rangle$, given by equation (2). Consequently, the equivalent width (EW) of the emission line,

$$
W \equiv \int \frac{\Delta I_{v}}{I_{v}\left(\lambda_{0}\right)} d \lambda
$$

is changed by a factor $\langle\mu\rangle / \mu$, namely,

$$
W=W_{0} \frac{\langle\mu\rangle}{\mu},
$$

where $W_{0}$ is the intrinsic EW of the unlensed quasar.

Even in the absence of lensing, quasars do not possess a single EW value in their emission lines but rather show a wide probability distribution of EW values (Francis 1992), which we define as $P\left(W_{0}\right)$ and model by a log-Gaussian form,

$$
P\left(W_{0}\right)=\frac{1}{\sqrt{2 \pi \sigma_{W}^{2}} W_{0}} \exp \left\{\frac{-\left[\ln \left(W_{0}\right)-\omega\right]^{2}}{2 \sigma_{W}^{2}}\right\} .
$$


The parameters $\omega$ and $\sigma_{W}$ obtain different values for different emission lines. The distribution in equation (9) is assumed to be observed in the absence of lensing. However, if the line of sight to a quasar passes at an impact parameter $x$ from the center of a galaxy with a MACHO fraction $\epsilon$, then its equivalent width is drawn from the distribution

$$
P(W, x, \epsilon)=\int_{1}^{\infty} d \mu p(\mu, x, \epsilon) P\left(\frac{\mu}{\langle\mu\rangle} W\right) \frac{\mu}{\langle\mu\rangle} .
$$

Figure 1 shows the probability distribution in equation (10) as a function of $x$, for $(a) \epsilon=0.3,(b) \epsilon=0.5$, and (c) $\epsilon=1$. The unlensed distribution was selected for the $\mathrm{Mg}$ II line, based on the data in Francis (1992). The microlensing signal is similar for other lines (Perna \& Loeb 1997). Naturally, the systematic distortion of the lensed distribution increases as $x$ decreases because of the corresponding increase in the surface density of MACHOs. However, the number of observed quasars declines as $x$ becomes smaller, and so the signal suffers from an increasing statistical noise in this limit. In order to properly evaluate the signal-tonoise ratio, we need to combine the above effects.

Let us consider a galaxy with luminosity $L$ at redshift $z$ and a population of background quasars with a flux greater than $S_{\mathrm{QSO}}$ and an average number per solid angle $N\left(>S_{\mathrm{QSO}}\right)$. We define $\theta_{s}$ to be the angle between the line of sight to a given quasar and the optical axis of the galaxy. The number of quasars within a ring of width $d \theta_{s}$ around $\theta_{s}$ is $N\left(>S_{\mathrm{QSO}}\right) 2 \pi \theta_{s} d \theta_{s}$. Using $\theta_{s}=\eta / D_{\text {os }}$, and defining $\theta_{0} \equiv$ $\xi_{0} / D_{\mathrm{ol}}$, this number can be expressed as

$$
\begin{aligned}
N\left(>S_{\mathrm{QSO}}\right) 2 \pi \frac{\eta d \eta}{D_{\mathrm{os}}^{2}} & =N\left(>S_{\mathrm{Qso}}\right) 2 \pi \frac{\xi_{0}^{2}}{D_{\mathrm{o}}^{2}} y d y \\
& =N\left(>S_{\mathrm{Qso}}, x, \epsilon\right) 2 \pi \theta_{0}^{2}(x-1) d x .
\end{aligned}
$$

The last step used the relation between $x$ and $y$ for a single image in the SIS model and incorporated amplification bias through the dependence of the local density of quasars with observed flux greater than $S_{\mathrm{Qso}}$ on $x$,

$$
N\left(>S_{\mathrm{QSO}}, x, \epsilon\right)=\frac{1}{\langle\mu(x)\rangle} \int_{1}^{\infty} d \mu p(\mu, x, \epsilon) N\left(>\frac{S_{\mathrm{QSO}}}{\mu}\right) .
$$

Equation (11) gives the number of quasars per unit solid angle inside a ring of radius $x$ and width $d x$ around the center of a single galaxy. To obtain the total number of quasars per solid angle which are seen within a distance $d x$ of $x$ of all galaxies up to a limiting flux $S_{\mathrm{gal}}$, one has to integrate over the galaxy distribution, both in luminosity and in redshift,

$$
\begin{aligned}
N(> & \left.S_{\mathrm{QSO}},>S_{\mathrm{gal}}, x, \epsilon\right) d x=\frac{2 \pi(x-1) d x}{\langle\mu(x)\rangle} \\
& \times \int_{1}^{\infty} d \mu p(\mu, x, \epsilon) N\left(>\frac{S_{\mathrm{QSO}}}{\mu}\right) \int_{0}^{z_{s}} d z\left|c \frac{d t}{d z}\right|(1+z)^{3} \\
& \times \int_{L_{\mathrm{gal}}}^{\infty} d L \phi_{G}(L) \xi_{0}^{2}(L, z) .
\end{aligned}
$$

Here we have implicitly assumed that the rings around different galaxies do not overlap. As shown later, the signal-tonoise ratio of the microlensing signature peaks at small values of $x$, where this assumption appears to be well satisfied. If $S_{\text {gal }}$ is the minimum flux needed to detect a galaxy, then $L_{\mathrm{gal}}$ in equation (13) is given by $L_{\mathrm{gal}}=4 \pi D_{L}^{2}(z) S_{\mathrm{gal}}$, with the luminosity distance $D_{L}(z)=(1+z)^{2} D_{\mathrm{ol}}(z)$.

\section{APPLICATIONS AND RESULTS}

Next, we apply the results from $\S 3$ to simulated data for a survey similar to SDSS. Let $S_{\mathrm{QSo}}$ and $S_{\mathrm{gal}}$ be the minimum fluxes needed for the detection of quasars and galaxies, respectively, and let $\Delta \Omega$ be the total solid angle surveyed on the sky. By definition, the overall (sky averaged) source count of quasars is not influenced by the amplification bias. Hence, the total number of detected quasars is given by $N_{\text {Tот }}=N\left(>S_{\mathrm{QSO}}\right) \Delta \Omega$, with $N(>S)$ from equation (5).

To simulate the results of real observations, we start by generating $N_{\text {тот }}$ random numbers drawn from the distribution

$$
P(x) \equiv \frac{1}{N\left(>S_{\mathrm{Qso}}\right)} N\left(>S_{\mathrm{QSo}},>S_{\mathrm{gal}}, x, \epsilon\right) .
$$

The value of the maximum $x, x_{\max }$, is fixed by the condition,

$$
\int_{0}^{x_{\max }} d x N\left(>S_{\mathrm{Qso}},>S_{\mathrm{gal}}, x, \epsilon\right)=N\left(>S_{\mathrm{QSO}}\right),
$$

to reflect the crude boundary beyond which the halos of foreground galaxies overlap. This approach assigns a halo cutoff radius that scales as a power law of $\sigma$, namely, $R_{\text {cutoff }}=\xi_{0} x_{\max }$, where $\xi_{0} \propto \sigma^{2}$. The precise value of $x_{\max }$ has little impact on our conclusions since the signal-tonoise ratio is high only for values of $x \leqslant x_{\max }$. Also notice that, in our actual calculation, the value of the lower limit of integration in equation (15) has been set to 2 to avoid the complications arising from multiple images. Since the fraction of quasars that are multiply imaged is very small $(\lesssim 1 \%)$, our statistical analysis is not affected by this assumption.

We have generated a source catalog at random based on equation (14), with equivalent widths $W$ chosen according to the probability distribution in equation (10). This data set is chosen to represent a mock realization of SDSS, for which we evaluate the signal-to-noise ratio of the microlensing signature, assuming a spectral resolution for the lines of $\sim 5 \AA$. Our analysis of this catalog involves several steps. First we bin all sources based on their equivalent width, $W_{i}$, and derive the "average" probability histogram $P_{\text {avg }}\left(W_{i}\right)$, which reflects equation (9) to within the statistical noise. We then bin the sources based on their lowest value of $x$ relative to a foreground galaxy. (Note that the association of a galaxy to a quasar based on the minimum $x$-value does not necessarily pick the closest galaxy to the quasar on the sky.) The equivalent widths of the quasars in each $x_{i}$ bin are then binned, and a $\chi^{2}$ analysis is performed to test the significance of the deviation of the data in each bin from the average distribution $P_{\text {avg }}\left(W_{i}\right)$. More specifically, we define $\chi^{2}=\sum_{i}\left[\left(\hat{n}_{i}-n_{i}\right)^{2} / n_{i}\right]$, where $\hat{n}_{i}$ is the number of quasars in the $\mathrm{EW}$ bin $i$ within a given $x$ bin and $n_{i}$ is the expected number according to the average distribution $P_{\text {avg }}$. The endpoint of each $x_{i}$ bin is varied (starting from the end of the previous $x_{i-1}$ bin) so as to minimize $P\left(\chi^{2}\right)$. This function is defined as the probability that a random set of data would provide a value of $\chi^{2}$ greater than the one obtained for the real data set. Clearly, the smaller $P\left(\chi^{2}\right)$, the more significant is the deviation of the data in each bin from $P_{\text {avg }}$.

Figures $2 a-2 c$ show $P\left(\chi^{2}\right)$ as a function of the binned $x$ for simulated sets of data with $z_{s}=3$, and magnitude detection 


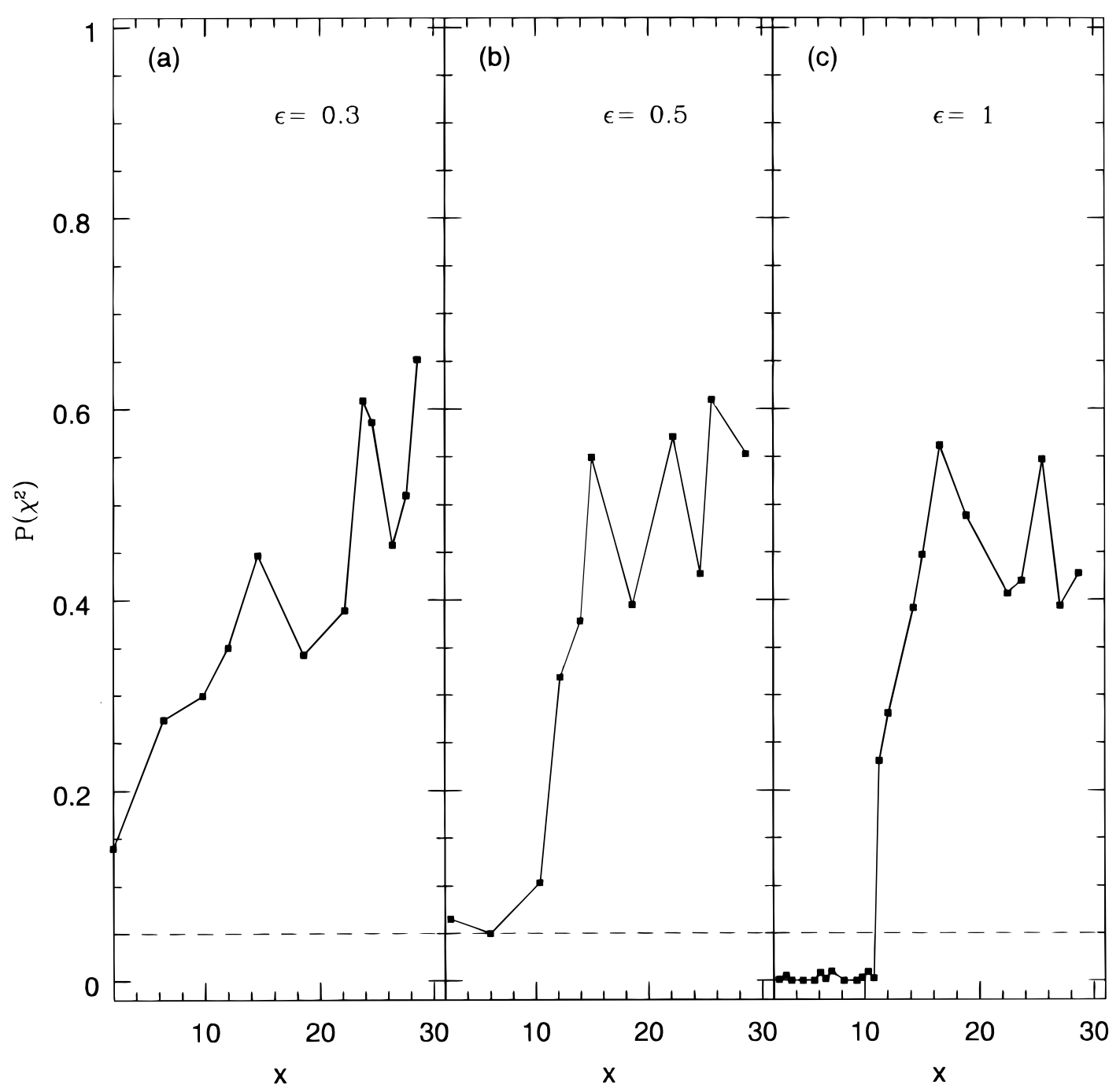

FIG. 2. $-\chi^{2}$ probability as a function of the binned $x$ for a simulated SDSS catalog, in which the equivalent widths and the quasar-galaxy separation $x$ of $\sim 10^{5}$ quasars are measured. The value of $P\left(\chi^{2}\right)$ indicates the probability that the EW data in each $x$ bin are described by the average distribution $P_{\text {avg }}\left(W_{i}\right)$, obtained by considering all the EWs, with no reference to quasar-galaxy separations. In $(a) \epsilon=0.3$, in $(b) \epsilon=0.5$, and in $(c) \epsilon=1$.

thresholds of $m_{\mathrm{gal}} \approx 22 \mathrm{R} \mathrm{mag}$ and $m_{\mathrm{Qso}} \approx 19.5 \mathrm{~B} \mathrm{mag}$, giving a total quasar count of $N_{\text {Tот }} \sim 10^{5}$, and a total number of galaxies $\sim 3 \times 10^{7}$, of the same order as expected for the forthcoming SDSS. Here we are using the limiting magnitude for the photometric sample of galaxies (larger by $\sim 1.5$ orders of magnitude than the spectroscopic sample), assuming that photometric redshift determination would be sufficiently accurate for estimating $\xi_{0}$ for each galaxy. In Figure $2 a$ the MACHO fraction is $\epsilon=0.3$; in Figure $2 b$, $\epsilon=0.5$; and in Figure $2 c, \epsilon=1$. The first case corresponds to the situation where the halo dark matter is dominated by a smooth dark matter component (e.g., elementary particles), and the last case to a situation where all the dark matter is in the form of MACHOs. The difference between the three panels is apparent. For a value of $\epsilon$ less than 0.5, $P\left(\chi^{2}\right)$ is of the order of several tenths, implying that the microlensing signal is not statistically significant. However, for $\epsilon \sim 0.5$, the value of $P\left(\chi^{2}\right)$ at small $x$ indicates that the EW distribution starts to become statistically different from $P_{\text {avg }}$ as a result of microlensing. The difference between these distributions is strong when the MACHO fraction is close to unity. Figure $2 c$ shows that the EWs of quasars, whose lines of sight intercept a galaxy at an impact param- eter $x \lesssim 10$ from its center, can be rejected as being drawn from the distribution $P_{\text {ave }}$ with a confidence level $\gtrsim 2 \sigma$ (the threshold level indicated by the dashed horizontal line). We have found this result to be robust using several realizations of the mock SDSS catalog.

The length scale associated with a given value of $x=\xi / \xi_{0}$ depends on the luminosity $L$ and redshift $z_{\ell}$ of the lensing galaxy, and on the source redshift $z_{s}$; for $z_{s}=3$,

$$
\xi_{0}=96 \frac{\left(L / L_{*}\right)^{0.4}}{\left(1+z_{\ell}\right)}\left[\frac{3}{2 \sqrt{1+z_{\ell}}}-\frac{1}{1+z_{\ell}}-\frac{1}{2}\right] \mathrm{kpc} .
$$

For example, an $L_{*}$ galaxy obtains $\xi_{0}=3.7 \mathrm{kpc}$ at $z_{\ell}=0.5$. Therefore, detection of a microlensing signal at $x \sim 10$ would probe galactic halos out to several tens of kiloparsecs, similar to the local microlensing searches. Since this scale extends well beyond the luminous cores of galaxies, our proposed method would sample the dark matter content of the intervening halos.

Note that due to the fact that the MACHO signal in Figure $2 c$ is statistically significant to $x=10$, the SDSS instruments should not introduce any significant selection bias against close quasar-galaxy pairs that might mask the 
microlensing signal. For an $L_{*}$ galaxy at $z=0.5$, the value of $x=10$ corresponds to an angular separation as large as $\sim 10^{\prime \prime}$.

\section{CONCLUSIONS}

We have shown that the MACHO fraction in extragalactic halos can be inferred from the distortion in the equivalent width distribution of background quasars as a function of their separation from foreground galaxies. This method could be used to test whether the properties of the Milky Way halo as inferred from local microlensing surveys are characteristic of other galaxies.

For the magnitude limit and survey area of SDSS, we find that if galactic halos are made of MACHOs, the ratio between the microlensing signal and the statistical rms noise is $\gtrsim 2$, extending to a halo radius of several tens of kiloparsecs (see Fig. 2). Larger surveys might be able to probe the signature of halos with a smaller mass fraction of MACHOs.

We thank the referee, Peter Schneider, for thoughtful comments on the manuscript, and Matthias Bartelmann for providing us with a computer routine that calculates the probability distribution of amplifications. This work was supported in part by NASA ATP grant NAG 5-3085 and the Harvard Milton fund (for A. L.) and by a fellowship from the University of Salerno, Italy (for R. P.).
Alcock, C., et al. 1996, ApJ, 486, 697

Ansari, R., et al. 1996, A\&A, 314, 94

Bartelmann, M., \& Schneider, P. 1990, A\&A, 239, 113

Canizares, C. R. 1982, ApJ, 263, 508

. 1984, in Quasars and Gravitational Lenses (Liège: Univ. Liège, Cointe-Ougree), 126

Crotts, A. P. S. 1996, in ASP Conf. Ser. 117, Dark and Visible Matter in Galaxies, ed. M. Persic \& P. Salucci (San Francisco: ASP), 289

Crotts, A. P. S., \& Tomaney, A. B. 1996, ApJ, 473, L87

Dalcanton, J. J., Canizares, C. R., Granados, A., \& Stocke, J. T. 1994, ApJ, 568,424

Francis, P. J. 1992, ApJ, 405, 119

Gould, A. 1996, ApJ, 470, 201

Gould, A., \& Miralda-Escudé, J. 1997, ApJ, 483, L13

Hartwick, F. D. A., \& Schade, D. 1990, ARA\&A, 28, 437

Hawkins, M. R. S., \& Taylor, A. N. 1997, ApJ, 482, L5

Laor, A. 1990, MNRAS, 246, 369

Lin, H., Kirshner, R. P., Shectman, S. A., Landi, S. D., Oemler, A., Tucker, D. L., \& Schechter, P. L. 1996, ApJ, 464, 60

Maoz, D. 1996, in IAU Colloq. 159, Emission Lines in Active Galaxies: New Methods and Techniques, ed. B. M. Peterson, F.-Z. Cheng, \& A. S. Wilson

\section{REFERENCES}

Marshall, H. L. 1985, ApJ, 299, 109

Netzer, H., \& Peterson, B. 1997, preprint (astro-ph/9706039)

Paczyński, B. 1986, ApJ, 304, 1

Perna, R., \& Loeb, A. 1997, ApJ, 489, 489

Peterson, B. M. 1993, PASP, 105, 247

Racine, R. 1991, AJ, 102, 454

Rauch, K. P., \& Blandford, R. D. 1991, ApJ, 381, L39

Schneider, P. 1987, A\&A, 179, 80

Schneider, P., Ehlers, J., \& Falco, E. E. 1992, Gravitational Lenses (Heidelberg: Springer)

Setti, G. 1984, in X-ray and UV emission from Active Galactic Nuclei, ed. W. Brinckmann \& J. Trümper (Garching)

Shaver, P. A., Wall, J. V., Kellermann, K. I., Jackson, C. A., \& Hawkins, M. R. S. 1996, Nature, 384, 439

Strauss, M. A., \& Willick, J. A. 1995, Phys. Rep., 261, 271

Tully, R. B., \& Fisher, J. R. 1977, A\&A, 54, 661

Wambsganss, J., Paczyński, B., \& Schneider, P. 1990, ApJ, 358, L33 